\title{
CONNECTION MATRICES AND TRANSITION MATRICES
}

\author{
CHRISTOPHER McCORD \\ Department of Mathematics, University of Cincinnati \\ P.O. Box 210025, Cincinnati, Ohio 45221-0025, U.S.A. \\ E-mail: chris.mccord@uc.edu \\ JAMES F. REINECK \\ Department of Mathematics, SUNY at Buffalo \\ Buffalo, New York 14214, U.S.A. \\ E-mail: reineck@math.buffalo.edu
}

\begin{abstract}
This paper is an introduction to connection and transition matrices in the Conley index theory for flows. Basic definitions and simple examples are discussed.

1. Introduction. A fundamental theorem of Conley says that in a flow there are two possible types of behavior for orbits in an isolated invariant set. A point can either be chain recurrent, or it can be on a connecting orbit from one chain recurrent piece to another chain recurrent piece. In the classical case of a gradient flow on a compact manifold, the chain recurrent pieces are the rest points, and Morse theory gives a relation between the Morse indices of the rest points and the topology of the manifold. Another way to look at this is to note that the Morse inequalities force connections between certain pairs of rest points in the gradient flow. Morse inequalities also apply to certain compact invariant subsets of the flow (which are denoted $M(I)$ below), so the topology of these subsets also forces the existence of connecting orbits.

In the more general case of a flow which is not necessarily gradient-like, instead of connections between rest points, the global topology forces connections between chain recurrent pieces of the flow. These pieces are called Morse sets, and the Morse index of the rest point is replaced by the homology Conley index in this more general setting. The Morse theoretic information can be encoded in a single matrix, called the connection matrix.
\end{abstract}

1991 Mathematics Subject Classification: Primary 58F25; Secondary 34C35.

Research of the first author supported in part by grants from the National Science Foundation and the Charles Phelps Taft Memorial Fund.

The paper is in final form and no version of it will be published elsewhere. 
For this algebraic encoding to be useful, it must be computable, and once computed, it must return new dynamical information. We will address both of these issues. In particular, we will consider how connection matrices can be applied to parameterized families of flows. This can be done in two different ways, each giving different types of information. By considering the stability of connection matrices under perturbation, we can detect stable connecting orbit structures. By studying the changes in connection matrices under perturbation, we can detect bifurcations in the connecting orbit structures. This last approach leads naturally to the introduction of transition matrices.

Most of the ideas discussed in this paper are originally due to Charles Conley, who was an inspiration to both of us, and to many others. The singular transition matrices introduced below were developed in the second author's thesis, which was done under Conley's direction.

In this note we consider only connection matrices for flows. Recently, Richeson has developed the ideas for continuous maps. See [13].

We assume that the reader is familiar with the notions of isolated invariant set, index pair, and Conley index. For references, see [2], [14], and [1]. If $S$ is an isolated invariant set, $C H(S)$ will denote its homology index, and we will use field coefficients for homology.

\section{Morse decompositions}

Definition 2.1. Let $S$ be an isolated invariant set. A subset $A \subset S$ is called an attractor in $S$ if there is an $S$-neighborhood $U$ of $A$ such that $\omega(U)=A$. A repeller is an attractor in the backward flow. If $A$ is an attractor in $S$, let $A^{*}=\{x \in S \mid \omega(x) \cap A=\emptyset\}$.

It is not hard to see that $A^{*}$ is a repeller. It is called the repeller dual to $A$, and the pair $\left(A, A^{*}\right)$ is called an attractor-repeller pair. Let $C\left(A^{*}, A\right)=\{x \in S \mid \omega(x) \subset$ $\left.A, \alpha(x) \subset A^{*}\right\}$ be the connecting orbits from $A^{*}$ to $A$. It can be shown that $S=$ $A \cup A^{*} \cup C\left(A^{*}, A\right)$.

The generalization of an attractor-repeller pair is a Morse decomposition. To define it, let $(P,<)$ be a finite partially ordered set, i.e. $p \nless p$ for all $p \in P$ and $<$ is transitive.

Definition 2.2. A Morse decomposition of an isolated invariant set $S$ is a collection $\{M(p) \mid p \in P\}$ of compact invariant subsets of $S$ such that if $x \in S \backslash \bigcup_{p \in P} M(p)$, then there are $p, q \in P, p<q$ such that $\omega(x) \subset M(p)$ and $\alpha(x) \subset M(q)$. The order $<$ on $P$ is called an admissible order.

The motivating example of a Morse decomposition is the rest points in a gradient flow. The actual definition of Morse decomposition generalizes this notion to flows which are not gradient-like. The chain recurrent behavior is contained in the $M_{p}$. The rest of $S$ lies on connecting orbits between the recurrent pieces.

Of all the admissible orders for a Morse decomposition, there is a minimal order (in the sense that it has the fewest relations among admissible orders), $<_{F}$, called the flow-defined partial order on $P$. This order is obtained by starting with $p<_{F} q$ if $C\left(M_{q}, M_{p}\right) \neq \emptyset$, then taking the transitive closure. All other admissible orders are obtained by adding relations to $<_{F}$. Of course, dynamically, one is interested in $<_{F}$, but other orders are important when one looks at perturbations of the flow, i.e. continuation. 
A Morse decomposition of $S$ gives rise to many attractor-repeller pairs. Recall that a set $I \subset P$ is an interval if $p, r \in I, q \in P$, and $p<q<r$ imply that $q \in I$. If $I$ is an interval, let

$$
M(I)=\bigcup_{i \in I} M(i) \cup \bigcup_{i, j \in I} C(M(j), M(i)) .
$$

One can show that $M(I)$ is an isolated invariant subset of $S$.

Definition 2.3. We say that $(I, J)$ is an adjacent pair of intervals if

1. $I \cup J$ is an interval, and

2. $i \in I, j \in J$ implies $j \nless i$.

Similarly, $(I, J, K)$ is an adjacent triple of intervals if

1. $I \cup J \cup K$ is an interval, and

2. $i \in I, j \in J$, and $k \in K$ implies $k \nless i, j$, and $j \nless i$.

We write $I J$ instead of $I \cup J$, and $I J K$ instead of $I \cup J \cup K$. It can be shown that if $(I, J)$ is an adjacent pair of intervals, then $(M(I), M(J))$ is an attractor-repeller pair in $M(I J)$

3. The flow-defined boundary map. In this section we introduce the flow-defined boundary map for an attractor-repeller pair. In classical Morse theory, when a critical level is passed, a $\lambda$-cell is attached where $\lambda$ is the Morse index of the critical point. To obtain the attaching map, one looks at the negative gradient flow on the unstable manifold of the critical point. The following construction generalizes this notion. Given an attractor-repeller pair, an isolating neighborhood of the repeller is attached, by flowing from the exit set, to a neighborhood of the attractor.

Definition 3.1. Let $S$ be an isolated invariant set, and let $\left(A, A^{*}\right)$ be an attractorrepeller pair in $S$. Then a triple $N_{0} \subset N_{1} \subset N_{2}$ of compact sets is called an index triple for $S$ if

1. $\left(N_{1}, N_{0}\right)$ is an index pair for $A$;

2. $\left(N_{2}, N_{0}\right)$ is an index pair for $S$;

3. $\left(N_{2}, N_{1}\right)$ is an index pair for $A^{*}$.

Now given an index triple $\left(N_{2}, N_{1}, N_{0}\right)$ for $\left(A, A^{*}\right)$, there is a short exact sequence of chain complexes

$$
0 \rightarrow C_{*}\left(N_{1}, N_{0}\right) \rightarrow C_{*}\left(N_{2}, N_{0}\right) \rightarrow C_{*}\left(N_{2}, N_{1}\right) \rightarrow 0
$$

which gives a long exact homology sequence

$$
\cdots \rightarrow H_{q}\left(N_{1}, N_{0}\right) \rightarrow H_{q}\left(N_{2}, N_{0}\right) \rightarrow H_{q}\left(N_{2}, N_{1}\right) \stackrel{\partial\left(A^{A} A^{*}\right)}{\rightarrow} H_{q-1}\left(N_{1}, N_{0}\right) \rightarrow \cdots .
$$

With an extra assumption on the homology, or an extra condition (regularity) on the index triple, which can always be satisfied, we have

$$
\cdots \rightarrow C H_{q}(A) \rightarrow C H_{q}(S) \rightarrow C H_{q}\left(A^{*}\right) \stackrel{\partial\left(A, A^{*}\right)}{\rightarrow} C H_{q-1}(A) \rightarrow \cdots .
$$


DEFinition 3.2. We call sequence (1) the long exact sequence of the attractor-repeller pair, and call the degree -1 map $\partial\left(A, A^{*}\right): C H_{*}\left(A^{*}\right) \rightarrow C H_{*}(A)$ the flow-defined boundary map.

The flow-defined boundary map is independent of the index triple in the following sense: if $\left(N_{2}, N_{1}, N_{0}\right)$ is an index triple which gives the map $\partial: H_{*}\left(N_{2}, N_{1}\right) \rightarrow H_{*}\left(N_{1}, N_{0}\right)$ and $\left(N_{2}^{\prime}, N_{1}^{\prime}, N_{0}^{\prime}\right)$ is another triple with $\partial^{\prime}: H_{*}\left(N_{2}^{\prime}, N_{1}^{\prime}\right) \rightarrow H_{*}\left(N_{1}^{\prime}, N_{0}^{\prime}\right)$, then there are flow-defined isomorphisms $h_{A^{*}}: H_{*}\left(N_{2}, N_{1}\right) \rightarrow H_{*}\left(N_{2}^{\prime}, N_{1}^{\prime}\right)$ and $h_{A}: H_{*}\left(N_{1}, N_{0}\right) \rightarrow$ $H_{*}\left(N_{1}^{\prime}, N_{0}^{\prime}\right)$ such that

commutes.

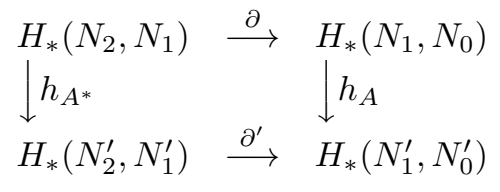

The importance of the flow-defined boundary map is given by the following.

Proposition 3.3. If $\partial\left(A, A^{*}\right) \neq 0$, then $C\left(A^{*}, A\right) \neq \emptyset$.

The idea of the proof is straightforward: if $C\left(A^{*}, A\right) \neq \emptyset$, then $S=A \cup A^{*}$, so $C H(S) \cong C H(A) \oplus C H\left(A^{*}\right)$. One then verifies that the long exact sequence (1) implies $\partial\left(A, A^{*}\right)=0$.

In the case of hyperbolic rest points and transverse connections, $\partial$ counts the number of connecting orbits "with orientation". In our examples, we will use $\mathbf{Z}_{2}$ coefficients for homology, so we state the following Theorem of McCord ([7]). $W^{s}$ and $W^{u}$ denote stable and unstable manifolds.

THEOREM 3.4. If $A$ is a hyperbolic rest point of index $k, A^{*}$ is a hyperbolic rest point of index $k+1$, and $W^{u}\left(A^{*}\right)$ intersects $W^{s}(A)$ transversely, then using $\mathbf{Z}_{2}$ coefficients, $\partial\left(A, A^{*}\right)$ counts the number of connecting orbits from $A^{*}$ to $A, \bmod 2$.

4. Connection matrices. Let $\{M(p) \mid p \in(P,<)\}$ be a Morse decomposition of an isolated invariant set $S$. If $p<q$ and $p$ and $q$ are adjacent, then there is a flow-defined boundary map $\partial(p, q): C H_{*}(q) \rightarrow C H_{*}(p)$ (where $C H_{*}(p)$ denotes $C H_{*}(M(p))$ ) of degree -1 which contains connection information by Proposition 3.3 and Theorem 3.4. Similarly, if $(I, J)$ is an adjacent pair of intervals, then there is a flow-defined boundary map $\partial(I, J): C H_{*}(J) \rightarrow C H_{*}(I)$. The maps $\partial(p, q)$ for $p, q \in P$ are determined by the global indices $C H(I)$. We will now see what restrictions the global dynamics places on the $\partial(p, q)$. In [4], Franzosa showed that it is possible to construct a filtration which simultaneously gives index pairs for all $M(I)$ where $I$ is an interval, and index triples for all attractor-repeller pairs $(M(I), M(J))$ in $M(I J)$ for adjacent pairs of intervals $(I, J)$. We assume that such a filtration has been chosen.

For $I \subset P$, let $C \Delta(I)=\bigoplus_{i \in I} C H(i)$. This is a graded vector space. A linear map $\Delta(P): C \Delta(P) \rightarrow C \Delta(P)$ is represented by a matrix

$$
[\Delta(p, q): C H(q) \rightarrow C H(p) \mid p, q \in P] .
$$

Definition 4.1. $\Delta(P)$ is upper triangular if $\Delta(p, q)=0$ if $p \nless q . \Delta(P)$ is a boundary map if each $\Delta(p, q)$ has degree -1 and $\Delta \circ \Delta=0$. 
Proposition 4.2. If $\Delta(P)$ is an upper triangular boundary map, then $\Delta(I)$ is an upper triangular boundary map for any interval $I \subset P$.

Proof. For any interval $I \subset P$, it is not difficult to see that there are intervals $J, K$ such that $J \cup I \cup K=P$ and $(J, I, K)$ is an adjacent triple of intervals. We can then decompose $\Delta$ as

$$
\Delta(P)=\left[\begin{array}{ccc}
\Delta(J) & \Delta(J, I) & \Delta(J, K) \\
0 & \Delta(I) & \Delta(I, K) \\
0 & 0 & \Delta(K)
\end{array}\right] .
$$

Taking the composition of the middle row and middle column gives $\Delta(I)^{2}=0$. Clearly $\Delta(I)$ inherits upper triangularity the degree -1 conditions, so $\Delta(I)$ is an upper triangular boundary map.

If $\Delta$ is an upper triangular boundary map, let $H \Delta(I)$ denote the homology of the chain complex $(C \Delta(I), \Delta(I))$. If $(I, J)$ is an adjacent pair of intervals, then with the obvious inclusion and projection maps there is a short exact sequence of chain complexes

$$
0 \rightarrow C \Delta(I) \rightarrow C \Delta(I J) \rightarrow C \Delta(J) \rightarrow 0
$$

which gives a long exact homology sequence

$$
\cdots \rightarrow H \Delta_{q}(A) \rightarrow H \Delta_{q}(S) \rightarrow H \Delta_{q}\left(A^{*}\right) \rightarrow H \Delta_{q-1}(A) \rightarrow \cdots .
$$

Notice that in the case $I=\{p\}, \Delta(\{p\})=0$ by upper triangularity, so $H \Delta(\{p\})=$ $C \Delta(\{p\})=C H(\{p\})$. We can now define the connection matrix.

Definition 4.3. An upper triangular boundary map $\Delta(P)$ is called a connection matrix for $\{M(p) \mid p \in(P,<)\}$ if for all intervals $I \subset P$, there are linear maps $\Phi(I)$ : $H \Delta(I) \rightarrow C H(I)$ satisfying

1. If $I=\{p\}$, then $\Phi(\{p\}): H \Delta(\{p\})=C H(\{p\}) \rightarrow C H(\{p\})$ is the identity.

2. For all adjacent pairs of intervals, the following diagram commutes

$$
\begin{aligned}
& \cdots \rightarrow H \Delta_{q}(I) \rightarrow H \Delta_{q}(I J) \rightarrow H \Delta_{q}(J) \rightarrow H \Delta_{q-1}(I) \rightarrow \cdots \\
& \downarrow \Phi(I) \quad \downarrow \Phi(I J) \quad \downarrow \Phi(J) \quad \downarrow \Phi(I) \\
& \cdots \rightarrow C H_{q}(I) \rightarrow C H_{q}(I J) \rightarrow C H_{q}(J) \rightarrow C H_{q-1}(I) \rightarrow \cdots
\end{aligned}
$$

where the top row is (2), and the bottom row is (1).

There are a couple of important facts which are immediate consequences of Definition 4.3 .

Proposition 4.4. $\Phi(I)$ is an isomorphism for all intervals $I$.

Proof. Induction and the 5-lemma.

Proposition 4.5. If $\Delta$ is a connection matrix and $p, q \in P$ are adjacent then $\Delta(p, q)$ $=\partial(p, q)$, the flow-defined boundary map.

The algebraic properties of $\Delta$ put restrictions on the $\partial(p, q)$. In applications, one often proceeds as follows. Some of the $\partial(p, q)$ are computed directly (by symmetry, or in an invariant subspace), and the algebraic conditions on $\Delta$ are used to determine the other maps. In this way, one proves the existence of connecting orbits. 
Let $\mathcal{C M}(<)$ denote the set of connection matrices with a given partial order $<$. Of course, one is most interested in the flow-defined order $<_{F}$. Other orders will be important in the continuation theory of connection matrices.

Franzosa ([5]) proved that connection matrices exist.

ThEOREM 4.6. Let $\{M(p) \mid p \in(P,<)\}$ be a Morse decomposition of an isolated invariant set $S$. Then $\mathcal{C} \mathcal{M}(<) \neq \emptyset$.

The relation between partial orders and connection matrices is straightforward. If $<_{1}$ and $<_{2}$ are admissible orders with $<_{1} \subset<_{2}$, (i.e. $p<_{1} q$ implies $p<_{2} q$ ), an interval $I \subset P$ in $<_{1}$ is also an interval in $<_{2}$, but the converse does not necessarily hold. Thus $<_{2}$ has more maps $\Phi(I)$, and hence more restrictions on $\Delta$. So we have the following.

Proposition 4.7. If $<_{1}$ and $<_{2}$ are admissible orders with $<_{1} \subset<_{2}$, then $\mathcal{C M}\left(<_{2}\right) \subset$ $\mathcal{C M}\left(<_{1}\right)$.

Corollary 4.8. If $<$ is an admissible order, then $\mathcal{C M}(<) \subset \mathcal{C M}\left(<_{F}\right)$.

5. Examples. In the examples we will use $\mathbf{Z}_{2}$ coefficients. Consider the flow in Figure 1 . Here $S$ consists of the the four rest points plus the connecting orbits between

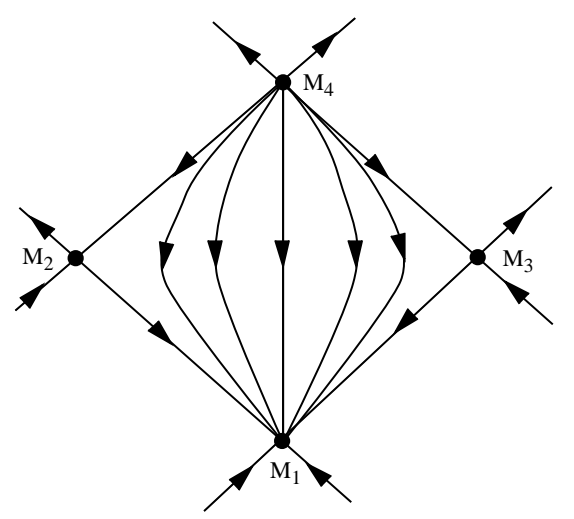

Fig. 1. $S$ is the box whose corners are the rest points

them. Using a rectangle which isolates $S$ it is not hard to see that $C H(S)=0$. The four rest points form a Morse decomposition with flow order $1<_{F} 2,3<_{F} 4$. We have $C H_{q}\left(M_{1}\right) \cong \mathbf{Z}_{2}$ if $q=0, C H_{q}\left(M_{2}\right) \cong C H_{q}\left(M_{3}\right) \cong \mathbf{Z}_{2}$ if $q=1, C H_{q}\left(M_{2}\right) \cong \mathbf{Z}_{2}$ if $q=2$, and all other homologies are 0 . Since $\Delta$ has degree -1 , the only possible nontrivial maps are $\partial(1,2), \partial(1,3), \partial(2,4)$, and $\partial(3,4)$. Since the corresponding sets are adjacent, all maps in $\Delta$ are flow-defined and by Theorem 3.4 , the maps are all isomorphisms. Thus

$$
\Delta=\begin{aligned}
& 1 \\
& 2 \\
& 3 \\
& 4
\end{aligned}\left(\begin{array}{llll}
0 & 1 & 1 & 0 \\
0 & 0 & 0 & 1 \\
0 & 0 & 0 & 1 \\
0 & 0 & 0 & 0
\end{array}\right) .
$$




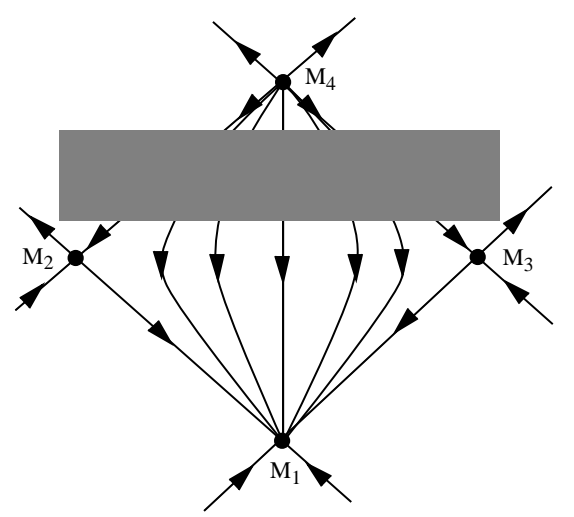

Fig. 2. Some missing information

This example is rather simple, but the power of the connection matrix is that one can deduce some of the entries if others are known. For example, suppose we had the information pictured in Figure 2, where the orbits in the shaded box are unknown. In particular, we know that that the four rest points are a Morse decomposition, that $C H(S)=0$, and that there are single connections from $M_{2}$ to $M_{1}$, and $M_{3}$ to $M_{1}$. It follows that $\partial(1,2)$ and $\partial(1,3)$ are isomorphisms, so $\Delta$ has the form

$$
\Delta=\begin{aligned}
& 1 \\
& 2 \\
& 3 \\
& 4
\end{aligned}\left(\begin{array}{llll}
0 & 1 & 1 & 0 \\
0 & 0 & 0 & a \\
0 & 0 & 0 & b \\
0 & 0 & 0 & 0
\end{array}\right),
$$

where $a, b$ are to be determined. Since $\Delta^{2}=0$, it follows that $a+b=0$ in $\mathbf{Z}_{2}$, i.e. $a=b$. Furthermore, since $C H(S)=0$, it follows that $\operatorname{dim}(\operatorname{ker} \Delta)-\operatorname{rank} \Delta=0$. Since $\operatorname{dim}(\operatorname{ker} \Delta)+\operatorname{rank} \Delta=4$, we have $\operatorname{rank} \Delta=2$. This implies $a=b=1$.

In the two previous example all of the maps in $\Delta$ were flow-defined boundary maps, so the connection matrix was unique. Nonuniqueness can arise when there are maps which are not flow defined. Consider the flow in Figure 3. There are two saddles with homology

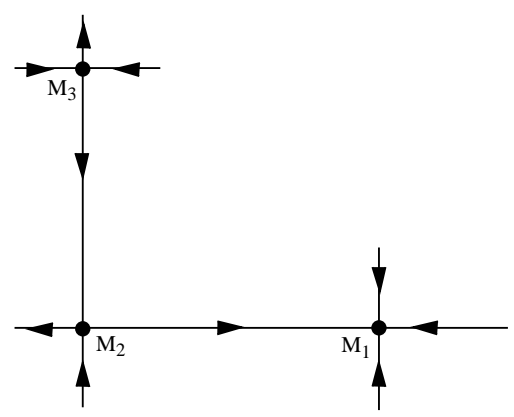

Fig. 3. A nontransverse connection 
in dimension one, and an attracting rest point with homology in dimension zero. $\mathrm{CH}(\mathrm{S})$ is one-dimensional, and the flow-defined order is $1<2<3$. There is a single connection from $M_{2}$ to $M_{1}$, so by Theorem $3.4 \Delta(1,2)=\partial(1,2)=1$. The map $\Delta(1,3)$ is not flow defined. We have

$$
\Delta=\begin{aligned}
& 1 \\
& 2 \\
& 3
\end{aligned}\left(\begin{array}{lll}
0 & 1 & a \\
0 & 0 & 0 \\
0 & 0 & 0
\end{array}\right) .
$$

It is easy to check that $\operatorname{dim} C H(S)=1$ implies $\operatorname{rank} \Delta=1$. In this example $\Delta^{2}=0$ and $\operatorname{rank} \Delta=1$ do not help determine $a$. In fact, one can construct isomorphisms $\Phi(I)$ for all intervals $I$ with either $a=0$ or $a=1$, so in this example there are two connection matrices. In the next section we shall see that the nonuniqueness is also a consequence of the continuation of connection matrices.

One can show that in the case that the Morse decomposition consists of hyperbolic rest points, and if $W^{u}\left(M_{p}\right)$ intersects $W^{s}\left(M_{q}\right)$ transversely for all $p, q \in P$, then every nonzero map in the connection matrix is flow-defined, so $\Delta$ is unique. In the case of a Morse-Smale flow, i.e. the Morse decomposition consists of hyperbolic rest points and periodic orbits, with transverse intersections, then nonuniqueness can occur. See [11].

6. Continuation. Suppose $\varphi_{\lambda}$ is a parameterized family of flows on $X$ with parameter space $\Lambda$. This gives a flow $\Phi$ on $X \times \Lambda$ via $\Phi_{t}(x, \lambda)=\left(\varphi_{t}^{\lambda} x, \lambda\right)$. Recall that $S^{\lambda}$, an isolated invariant set in $X$ for $\varphi^{\lambda}$, and $S^{\mu}$, an isolated invariant set in $X$ for $\varphi^{\mu}$, are related by continuation if there is an isolated invariant set $S \subset X \times \Lambda$ such that $S^{\lambda}=S \cap(X \times\{\lambda\})$ and $S^{\mu}=S \cap(X \times\{\mu\})$. Sets which are related by continuation have the same index. Indeed, there is a flow-defined isomorphism $F_{\mu \lambda}: C H\left(S^{\lambda}\right) \rightarrow C H\left(S^{\mu}\right)$. Recall that if $N$ is an isolating neighborhood for $\varphi^{\lambda}$ and $S^{\lambda}=\operatorname{Inv}\left(N, \varphi^{\lambda}\right)$, then for $\mu$ sufficiently close to $\lambda, N$ is an isolating neighborhood for $\varphi^{\mu}$ and $S^{\mu}=\operatorname{Inv}\left(N, \varphi^{\mu}\right)$ is related to $S^{\lambda}$ by continuation. Similarly if $\left\{M_{p}^{\lambda} \mid p \in P\right\}$ is a Morse decomposition of $S^{\lambda}$, then for $\mu$ sufficiently close to $\lambda$, there will be a Morse decomposition $\left\{M_{p}^{\mu} \mid p \in P\right\}$ such that for each $p, M_{p}^{\mu}$ and $M_{p}^{\lambda}$ are related by continuation. Thus there is a continuation isomorphism between homology indices, so we may regard a connection matrix for the $M_{p}^{\lambda}$ as acting on the homology indices of the $M_{p}^{\mu}$. So it makes sense to ask if there is containment of the set of connection matrices for $M_{p}^{\mu}$ in the set of connection matrices for $M_{p}^{\lambda}$. We shall see that this is the case.

It is important to consider how the flow order changes under perturbation. One can show that a small perturbation can break existing connections between sets in a Morse decomposition, e.g. the saddle-saddle connection in Figure 3 can be broken, but new connections cannot be created by small perturbations. If $<_{\lambda}$ denotes the flow-defined order on $\left\{M_{p}^{\lambda} \mid p \in P\right\}$, then for $\mu$ sufficiently close to $\lambda,<_{\lambda}$ will be an admissible order for the Morse decomposition $\left\{M_{p}^{\mu} \mid p \in P\right\}$. However, $<_{\lambda}$ may not necessarily be the flow order for the $M_{p}^{\mu}$. To see this, consider the following example. Here we have perturbed the flow from Figure 3 in two ways. The original flow order in Figure 3 was $1<_{\lambda} 2<_{\lambda} 3$. In the perturbation on the left of Figure 4 the flow order is $1<2$, and in the perturbation 

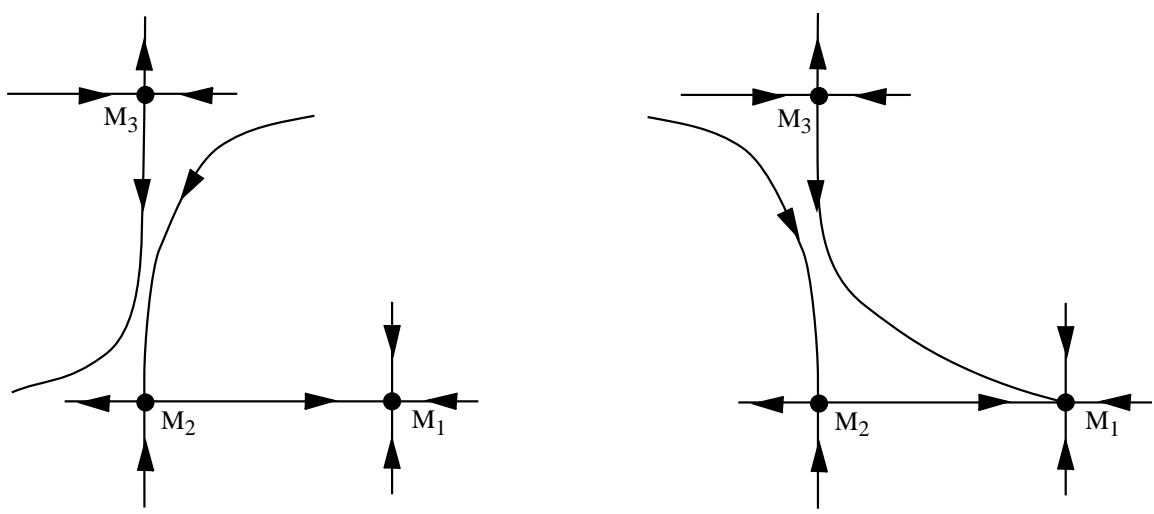

Fig. 4. Two perturbations of the saddle-saddle connection of Figure 3

on the right, the flow order is $1<2,1<3$. Notice that $1<2<3$ is an admissible order for both perturbations.

Now fix a $\lambda$ and suppose $S^{\lambda}$ is an isolated invariant set with Morse decomposition $\left\{M_{p}^{\lambda} \mid p \in P\right\}$. Let $\mathcal{C M}\left(<_{\lambda}\right)$ denote the set of connection matrices with the flow order on $P$. Assume $\mu$ is sufficiently close to $\lambda$ so that there is a Morse decomposition $\left\{M_{p}^{\mu} \mid p \in P\right\}$ such that for each $p, M_{p}^{\mu}$ and $M_{p}^{\lambda}$ are related by continuation. For each $\mu$, let $\mathcal{C} \mathcal{M}\left(<_{\mu}\right)$ denote the set of connection matrices for the $M_{p}^{\mu}$ with the flow order.

THeOREM 6.1. If $\mu$ is sufficiently close to $\lambda$, then $\mathcal{C M}\left(<_{\mu}\right) \subset \mathcal{C M}\left(<_{\lambda}\right)$.

The proof of this result is straightforward. If $\mu$ is close to $\lambda$, then $<_{\lambda}$ is an admissible order for $\left\{M_{p}^{\mu} \mid p \in P\right\}$. So by Corollary $4.8, \mathcal{C M}\left(<_{\mu}\right) \subset \mathcal{C M}\left(<_{\lambda}\right)$.

We can apply Theorem 6.1 to show that there is not a unique connection matrix for the flow in Figure 3. An arbitrarily small perturbation of the flow can give either of the phase portraits in Figure 4 . In the portrait on the left, $1<2$ is the only relation, $\Delta(1,2)=$ $\partial(1,2)=1$, all other entries are 0 , so $\Delta=\left(\begin{array}{lll}0 & 0 & 1 \\ 0 & 0 & 0 \\ 0 & 0 & 0\end{array}\right)$. In the portrait on the right both $\Delta(1,2)$ and $\Delta(1,3)$ are flow-defined isomorphisms, so $\Delta=\left(\begin{array}{lll}0 & 1 & 1 \\ 0 & 0 & 0 \\ 0 & 0 & 0\end{array}\right)$. Since both of these connection matrices can be realized by an arbitraryily small perturbation of Figure 4, Theorem 6.1 says that both of these matrices are connection matrices for the original flow with the saddle-saddle connection.

7. Transition matrices. Connection matrices give information about the connecting orbit structure in a single flow. For a parameterized family of flows, the continuation of connection matrices described in the previous section reveals two things:

- Connection matrices detect connecting orbit structures which persist under perturbation (codimension 0 connections). 
- Changes in the connection matrices detect changes in the connecting orbit structure (codimension 1 connections).

But the mere fact that the collection of connection matrices changes does not reveal what changes have taken place in the connecting orbits. To obtain finer information, we must study the changes in the connection matrices more systematically. The devices introduced to do so are known as transition matrices. Actually, there are two different constructions of transition matrices: topological transition matrices and singular transition matrices. We will consider each in turn.

The setting for both is the same. Fix a parameterized family of flows $\varphi^{\lambda}$ on $X$ with parameter space $\Lambda$. Suppose that $S$ is an isolated invariant set in $X$ which continues across $\Lambda$, and $\left\{M_{p} \mid p \in P\right\}$ is a Morse decomposition that also continues across $\Lambda$. That is, for every $\lambda \in \Lambda,\left\{M_{p}^{\lambda} \mid p \in P\right\}$ is a Morse decomposition of $S^{\lambda}$.

7.1. Topological transition matrices. We begin with the simplest situation. Within $\Lambda$, let $\Lambda^{\prime}=\left\{\lambda \in \Lambda \mid S^{\lambda}=\sqcup_{p \in P} M_{p}^{\lambda}\right\}$. That is, $\Lambda_{0}$ is the set of parameter values for which no connecting orbits occur. Clearly, the only possible connection matrix for $\left\{M_{p}^{\lambda} \mid p \in\right.$ $\left.P, \lambda \in \Lambda^{\prime}\right\}$ is the zero matrix, so the continuation of connection matrices cannot detect any codimension 0 connecting orbits. But it is possible to detect codimension 1 connecting orbit structures.

Suppose $\lambda, \mu \in \Lambda^{\prime}$. Since $S^{\lambda}=\sqcup_{p \in P} M_{p}^{\lambda}$, there is a canonical isomorphism $\Phi_{\lambda}(P)$ : $C \Delta(P) \rightarrow C H\left(S^{\lambda}\right)$. Further, since each $S_{p}^{\lambda}$ is related by continuation to $S_{p}^{\mu}$ and $S^{\lambda}$ is related by continuation to $S^{\mu}$, there are isomorphisms $F \Delta_{\mu \lambda}(P)=\bigoplus_{p \in P} F_{\mu \lambda}: C \Delta_{\lambda}(P) \rightarrow$ $C \Delta_{\mu}(P)$ and $F_{\lambda \mu}: C H\left(S^{\lambda}\right) \rightarrow C H\left(S^{\mu}\right)$. These can be composed into a diagram

$$
\begin{array}{lcc}
C \Delta_{\lambda}(P) & \stackrel{F \Delta_{\mu, \lambda}(P)}{\longrightarrow} & C \Delta_{\mu}(P) \\
\downarrow \Phi_{\lambda}(P) & & \\
C H\left(S^{\lambda}\right) & \stackrel{F_{\mu, \lambda}}{\longrightarrow} & C \Phi_{\mu}(P) \\
& & \left.C S_{\mu}\right) .
\end{array}
$$

This diagram may not commute! Moreover, that failure of commutativity can be quantified, and used to detect changes in the connecting orbit structure. To do so, it is convenient to think of the continuation isomorphisms $F \Delta_{\mu, \lambda}(P)$ as fixing a set of coordinates on $C \Delta(P)$ that continues across $\Lambda$. Since $F \Delta_{\nu \mu}(P) F \Delta_{\mu \lambda}=F \Delta_{\nu \lambda}$, this process is well-defined. Then, let $T_{\mu \lambda}(P)=\Phi_{\mu}^{-1}(P) F_{\mu \lambda} \Phi_{l}(P) . T_{\mu \lambda}$ is the topological transition matrix from $\lambda$ to $\mu$.

THEOREM 7.1. Topological transition matrices have the following properties:

- If $\lambda, \mu, \nu \in \Lambda^{\prime}$, then $T_{\nu \mu} T_{\mu \lambda}=T_{\nu \lambda}$.

- If $\lambda, \mu$ lie in the same path component of $\Lambda^{\prime}$, then $T_{\mu \lambda}=i d$

- It follows from these that $T_{\lambda \mu}=T_{\mu \lambda}^{-1}$.

- If $p<q$, then $T_{\mu \lambda}(q, p): C H\left(S_{p}^{\lambda}\right) \rightarrow C H\left(S_{q}^{\mu}\right)$ is trivial.

- If $I$ is an interval in $P$, then $T_{\mu \lambda}(I)$ is a submatrix of $T_{\lambda \mu}(P)$.

Thus $T_{\mu \lambda}$ is upper triangular with respect to $<$, with $i d$ on the diagonal. In particular, if $T_{\mu \lambda}(p, q) \neq 0$, then $p<q$ in the flow-defined order along any path connecting $\lambda$ and $\mu$ in $\Lambda$. That is, along every path from $\lambda$ to $\mu$ in $\Lambda$ there must be a sequence of connecting orbits 


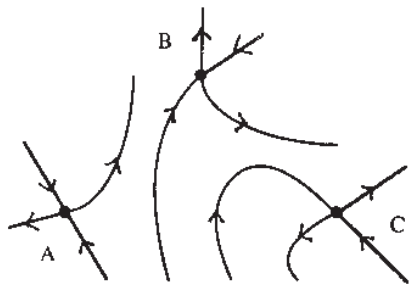

$\lambda_{0}$

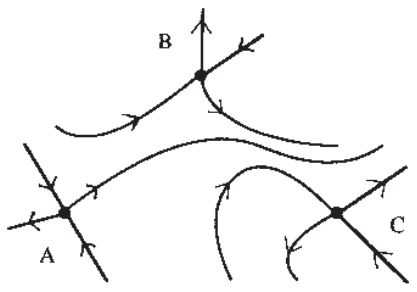

$\lambda_{3}$

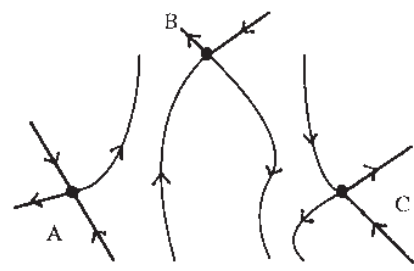

$\lambda_{1}$

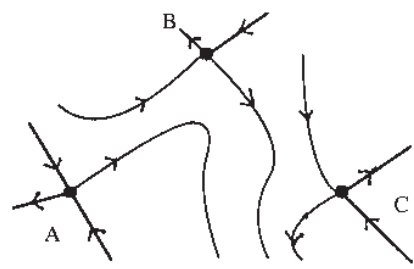

$\lambda_{3}$

Fig. 5. A two-parameter family of flows

(possibly at different parameter values) between $M_{q}$ and $M_{p}$. Moreover, since topological transition matrices are degree 0 isomorphisms, $T_{\mu \lambda}(p, q)$ can only be non-zero if there is some $k$ for which $C H_{k}\left(S_{p}\right)$ and $C H_{k}\left(S_{q}\right)$ are both non-zero. That is, the connecting orbits changes detected by topological transition matrices must be codimension 1 , degree 0 connections.

This form of transition matrix has the obvious disadvantage that it can only be employed when there are parameter values with no connecting orbits; and even then can only be used to detect changes between such parameter values. On the other hand, the relatively straightforward way in which they are defined, and the ability to compute transition matrices via compositions and inverses, make them easy to compute.

Topological transition matrices were introduced in [8] to analyse two-parameter families of flows. An archetypical situation is shown in Figure 5:

- For every parameter value, there are three Morse sets $M_{A}, M_{B}, M_{C}$, each with index $\Sigma^{n}$, the pointed $n$-sphere. The ordering $A<B<C$ is admissible across the parameter space.

- For an open dense set of parameters, there are no connections between the Morse sets. However, there is a curve $\Gamma_{B A}$, and a transverse curve $\Gamma_{C B}$, along which $B \rightarrow A$ and $C \rightarrow B$ connections occur.

Let $T_{i j}$ denote the topological transition matrix from $\lambda_{j}$ to $\lambda_{i}$. If there are no connections between $M_{A}, M_{B}$ and $M_{C}$ except the $B \rightarrow A$ connections along $\Gamma_{B A}$ and the $C \rightarrow B$ connections along $\Gamma_{C B}$, then

$$
T_{10}=T_{32}=\left(\begin{array}{ccc}
1 & 0 & 0 \\
0 & 1 & \alpha \\
0 & 0 & 1
\end{array}\right)
$$


and

$$
T_{20}=T_{31}=\left(\begin{array}{ccc}
1 & \beta & 0 \\
0 & 1 & 0 \\
0 & 0 & 1
\end{array}\right)
$$

with $\alpha$ and $\beta$ non-zero. The composition rule for transition matrices implies that $T_{32} \circ$ $T_{20}=T_{30}=T_{31} \circ T_{10}$. But it is a simple matter to verify that $T_{32} \circ T_{20} \neq T_{31} \circ T_{10}$. Thus, there must be some other connecting orbits. In particular, if there are no connecting orbits from $M_{C}$ to $M_{B}$ or $M_{A}$ along $\Gamma_{B A}$, and no connecting orbits from $M_{C}$ or $M_{B}$ to $M_{A}$ along $\Gamma_{C B}$, then there must be an arc $\Gamma_{C A}$ emanating from $\Gamma_{C B} \cap \Gamma_{B A}$, with connections $C \rightarrow A$ for all $\lambda \in \Gamma_{C A}$.

7.2. Singular transition matrices. The other approach to transition matrices is the singular transition matrix of Reineck. This is actually the older approach. The adjective singular was attached afterwards by McCord and Mischaikow to contrast it to the topological transition matrices they introduced. Though a later addition, the term is apt, for Reineck's transition matrices are defined by a limiting process which involves an essential loss of isolation in the limit.

Singular transition matrices require a $C^{1}$ family of flows, and work most naturally for a one parameter family of flows $\dot{x}=g(x, \lambda)$ for $\lambda \in[0,1]$. We will find it convenient to extend the parameter range slightly, and assume that $g(x, s)$ is defined for $s \in\left[-\frac{1}{2}, \frac{3}{2}\right]$. From this, we define a flow on $X \times\left[-\frac{1}{2}, \frac{3}{2}\right]$ by

$$
\begin{aligned}
\dot{x} & =g(x, s) \\
\dot{s} & =\epsilon s(s-1)
\end{aligned}
$$

If $N$ isolates $\bigcup_{-\frac{1}{2} \leq \lambda \leq \frac{3}{2}} S^{\lambda}$ in $X \times\left[-\frac{1}{2}, \frac{3}{2}\right]$ when $\epsilon=0$, then it also isolates some $S_{\epsilon}$ for $\epsilon>0$. Moreover, that $S_{\epsilon}$ has a Morse decomposition consisting of $\left\{M_{p}^{0}\right\}_{p \in P} \cup\left\{M_{p}^{1}\right\}_{p \in P}$. Of course, that collection is not a Morse decomposition at $\epsilon=0$ (hence the term singular). The index set for this Morse decomposition is $P \times\{0,1\}$.

This index set admits several different partial orders, and it will be crucial to our interpretation of transition matrices to understand the relation between these orders. First, if $<$ is the flow-defined partial order on $P$ across [0,1] (i.e. $p$ and $q$ are adjacent if and only if there is a connection from $S_{q}^{t}$ to $S_{p}^{t}$ for some $\left.t \in[0,1]\right)$, then we can define the product partial order $<_{p}$ on $P \times\{0,1\}$ by $(p, i)<(q, j)$ if either $p=q, i<j$, or $p<q, i \leq j$.

On the other hand, for each $\epsilon$, the drift flow has a flow-defined order $<_{\epsilon}$. Let $<_{d}$ be the inverse limit of these partial orders. Namely, $(p, i)<_{d}(q, j)$ if there exists a sequence $\epsilon_{n} \rightarrow 0$ such that $(p, i)<_{\epsilon_{n}}(q, j)$ for every $n$. In other words, $(p, i)<(q, j)$ in the drift partial order if there is a connection (or sequence of connections) from $M_{q}^{j}$ to $M_{p}^{i}$ in every open family of slow drift flows. In particular, if $(p, i)<_{\epsilon}(q, j)$ for all small $\epsilon$, then $(p, i)<_{d}(q, j)$.

Lemma 7.2. The flow-defined partial order $<$ on $P \times\{0,1\}$ refines the drift partial order $<_{d}$.

The upshot of this is that if $(p, 0)<_{\epsilon}(q, 1)$ for all small $\epsilon$, then there is some sequence $t_{n} \in[0,1]$ and $p_{n} \in P$ with $p_{0}=p, p_{N}=q$ and $M_{p_{i}}^{t_{i}}$ and $M_{p_{i+1}}^{t_{i}}$ adjacent in the $t_{i}$-flow. 
The drift flow does more than just make $\left\{M_{p}^{0}\right\}_{p \in P} \cup\left\{M_{p}^{1}\right\}_{p \in P}$ a Morse decomposition. For every $p \in P,(p, 0)$ and $(p, 1)$ are adjacent. In the flow on the interval $\left[-\frac{1}{2}, \frac{3}{2}\right], 0$ is an attracting fixed point, 1 is a repelling fixed point and $[0,1]$ is an isolated invariant set with index $\overline{0}$. Thus the product formula for the index implies that

for all $p \in P$.

$$
\begin{array}{cccc}
C H_{k}(M(p, 0)) & \cong & C H_{k}\left(M_{p}\right) \\
C H_{k}(M\{(p, 0),(p, 1)\}) & = & 0 \\
C H_{k}(M(p, 1)) & \cong & C H_{k-1}\left(M_{p}\right)
\end{array}
$$

The point is, there are connection matrices for this Morse decomposition, just as for any other. But the special nature of this Morse decomposition casts that connection matrix in a particular form, and gives a particular interpretation to the connection information it produces. First, the matrix is defined on $C_{k} \Delta(P \times\{0,1\}) \cong C_{k} \Delta(P \times\{0\}) \oplus$ $C_{k-1} \Delta(P \times\{1\})$ The matrix then has the form

$$
\Delta=\left(\begin{array}{ll}
\Delta_{0} & T \\
0 & \Delta_{1}
\end{array}\right)
$$

with $\Delta_{0}$ and $\Delta_{1}$ connection matrices on $P \times\{0\}$ and $P \times\{1\}$ respectively (actually, $\Delta_{1}$ is the suspension of a connection matrix on $C \Delta(P \times\{1\}))$. The matrix $T$ can be interpreted as a singular transition matrix $T: C_{k} \Delta(P \times\{1\}) \rightarrow C_{k} \Delta(P \times\{0\})$.

Since connection matrices are not unique, transition matrices are not unique. Some elements of $T$ are uniquely defined, however. For every $p \in P$, the pair $(p, 0),(p, 1)$ is adjacent. The homology Conley indices of $(p, 0),(p, 1)$ and the interval $\{(p, 0),(p, 1)\}$ imply that the connection homomorphism of this adjacent pair is the identity map. And, if $p \nless q$, then $(p, 0) \nless(q, 1)$, and $T_{q p}=0$. That is, $T$ has the form

$$
\left(\begin{array}{ccc}
i d & * & * \\
0 & \ddots & * \\
0 & 0 & i d
\end{array}\right)
$$

That is, it is upper-triangular with respect to $<$, with $i d$ on the diagonal.

COROLlaRY 7.3. A singular transition matrix $T$ is an upper-triangular isomorphism with respect to the drift partial order $<_{d}$. The diagonal entries of $T$ are given by $T(p, p)=$ $F_{\alpha^{-1}}(p) \circ \Sigma^{-1}(p)$.

Consider the family of Figure 4, with the two parameter values shown taken as $\lambda=0$ and $\lambda=1$. The partial order $2>1<3$ continues across the interval, but is not necessarily the flow-defined order across the interval. Must there be a connection $2<3$ for some parameter value between 0 and 1? If so, is it detectable by a transition matrix? The connection matrices $\Delta_{0}$ for $\lambda_{0}$ and $\Delta_{1}$ for $\lambda_{1}$ are unique, so any connection matrix for the drift flow on the product must have the form

$$
\Delta=\left(\begin{array}{rrrrrr}
0 & 1 & 0 & 1 & 0 & 0 \\
0 & 0 & 0 & 0 & 1 & a \\
0 & 0 & 0 & 0 & 0 & 1 \\
0 & 0 & 0 & 0 & -1 & -1 \\
0 & 0 & 0 & 0 & 0 & 0 \\
0 & 0 & 0 & 0 & 0 & 0
\end{array}\right)
$$


The condition $\Delta^{2}=0$ forces $a=1$. Thus the unique transition matrix is

$$
T=\left(\begin{array}{lll}
1 & 0 & 0 \\
0 & 1 & 1 \\
0 & 0 & 1
\end{array}\right)
$$

for any one-parameter family of flows connecting the two flows shown.

If $\left\{M_{p} \mid p \in P\right\}$ is a Morse decomposition that continues across $\Lambda$, let $\mathcal{P}(\Lambda)$ denote the path space on $\Lambda$. For every $\alpha \in \mathcal{P}(\Lambda)$, there is a product flow on $X \times\left[-\frac{1}{2}, \frac{3}{2}\right]$ defined by

$$
\begin{aligned}
& \dot{x}=g(x, \alpha(s)) \\
& \dot{s}=\epsilon s(s-1)
\end{aligned}
$$

Let $\mathcal{C M}\left(<_{\alpha}\right)$ denote the set of connection matrices on $P \times\{0,1\}$ for this path, and let $\mathcal{T}\left(<_{\alpha}\right)$ denote the corresponding set of transition matrices. It is natural to ask about the dependence of $\mathcal{T}\left(<_{\alpha}\right)$ on the path $\alpha$. Some information comes from applying the continuation results to $\mathcal{C M}\left(<_{\alpha}\right)$.

TheOrem 7.4. If $\beta$ is sufficiently close to $\alpha$ in $\mathcal{P}(\Lambda)$, then $\mathcal{T}\left(<_{\beta}\right) \subset \mathcal{T}\left(<_{\alpha}\right)$.

There are other, equally natural questions that are currently unanswered:

- What is the relationship between $\mathcal{T}(\alpha)$ and $\mathcal{T}\left(\alpha^{-1}\right)$ ?

- If $\alpha_{1}$ and $\alpha_{2}$ are paths with $\alpha_{1}(1)=\alpha_{2}(0)$, what is the relationship between $\mathcal{T}\left(<_{\alpha_{1}}\right), \mathcal{T}\left(<_{\alpha_{2}}\right)$ and $\mathcal{T}\left(<_{\alpha_{1} * \alpha_{2}}\right)$ ?

These questions are open in general, but are resolved in one case. When the set $\Lambda^{\prime}$ defined in $\S 7.1$ is non-empty, it is possible to define both topological and singular transition matrices. In this setting, the two definitions agree [8]. That is, the topological transition matrix is the unique singular transition matrix. The properties of topological transition matrices stated in Theorem 7.1 then become properties of singular transition matrices.

This property is exploited in [6] to prove the existence of infinitely many connecting orbits in a one-parameter family of differential equations in $R^{2}$ with slow parameter drift

$$
\begin{aligned}
\dot{u} & =g(u, \lambda, \epsilon) & & u \in R^{2} \\
\dot{\lambda} & =\epsilon g(\lambda) & & \lambda \in R
\end{aligned}
$$

with $g(\lambda)>0$ for $|\lambda|<1$ and $g(\lambda)<0$ for $|\lambda|>1$. When $\epsilon<0$, the drift is from $\lambda=1$ to $\lambda=-1$; the drift is reversed when $\epsilon=0$. As with any singular perturbation problem, the approach is to make assumptions about the behavior of the system at $\epsilon=0$, then determine what behavior will be observed when $\epsilon \neq 0$.

The hypotheses guarantee that the $\epsilon=0$ system has exactly two rest points for each $\lambda$ : one a saddle for all $\lambda$; the other an attractor for $\lambda<0$ and a repeller for $\lambda>0$. Further, $\lambda= \pm 1$, there is a unique transverse intersection of the stable and unstable manifolds. These solutions form an invariant, normally hyperbolic curve. for $\epsilon$ near zero, this curve persists and is further assumed to contain two hyperbolic equilibria. Under these hypotheses, it is shown that for a perturbation $\epsilon<0$, the number of heteroclinic orbits is at least two, but is bounded. On the other hand, for $\epsilon>0$, there are infinitely many heteroclinic orbits. 
The existence of the finite number of connections for $\epsilon<0$ comes from directly computing the singular transition matrix $T_{-}$. At $\epsilon=0$, this is a topological transition matrix, and for $\epsilon>0$, the unique singular transition matrix must be $T_{+}=T_{-}^{-1}$. It is from the structure of $T_{+}$that they deduce the existence of infinitely many connections.

For a straightforward application involving a family of flows arising from ecological models, see [12]. In this paper families of flows in the plane are considered with Morse decompositions consisting of hyperbolic rest points. Some connections can be deduced using symmetry, and the algebraic conditions discussed in section 4 are used to produce a list of connection matrices. These matrices correspond to flows without saddle-saddle connections. Transition matrices are computed algebraically to give information about saddle-saddle connections which can occur.

\section{References}

[1] L. Arnold, C. Jones, K. Mischaikow and G. Raugel, Dynamical Systems Montecatini Terme 1994, R. Johnson, ed., Lect. Notes Math. 1609, Springer, 1995.

[2] C. Conley, Isolated Invariant Sets and the Morse Index, CBMS Reg. Conf. Ser. in Math., 38, AMS, Providence, 1978.

[3] C. Conley, A qualitative singular perturbation theorem, Global Theory of Dynamical Systems, (eds. Z. Nitecki and C. Robinson), Lecture Notes in Math. 819, Springer-Verlag 1980, 65-89

[4] R. Franzosa, Index filtrations and the homology index braid for partially ordered Morse decompositions, Trans. AMS 298 (1986), 193-213.

[5] R. Franzosa, The connection matrix theory for Morse decompositions, Trans. AMS 311 (1989), 781-803.

[6] H. Kokubu, K. Mischaikow and H. OKA, Existence of infinitely many connecting orbits in a singularly perturbed ordinary differential equation, Nonlinearity 9 (1996), 1263-1280.

[7] C. MCCORD, The connection map for attractor-repeller pairs, Trans. AMS 308 (1988), 195-203.

[8] C. MCCord and K. Mischaikow, Connected simple systems, transition matrices and heteroclinic bifurcations, Trans. AMS 333 (1992), 397-422.

[9] C. MCCord and K. Mischaikow, Equivalence of topological and singular transition matrices in the Conley index, Mich. Math. J. 42 (1995), 387-414.

[10] J. Reineck, Connecting orbits in one-parameter families of flows, Erg. Thy. \& Dyn. Sys. $8^{*}$ (1988), 359-374.

[11] J. REINECK, The connection matrix in Morse-Smale flows, Trans. AMS 322 (1990), 523545 .

[12] J. Reineck, A connection matrix analysis of ecological models, Nonlin. Anal. 17 (1991), 361-384.

[13] Connection matrix pairs for the discrete Conley index, preprint. Available at http:/www.math.nwu.edu/ richeson.

[14] D. Salamon, Connected Simple Systems and the Conley index of isolated invariant sets, Trans. AMS 291 (1985), 1-41.

[15] J. Smoller, Shock Waves and Reaction-Diffusion Equations, Springer-Verlag, 1980.

[16] E. Spanier, Algebraic Topology, McGraw Hill, 1966, Springer-Verlag, New York, 1982. 